
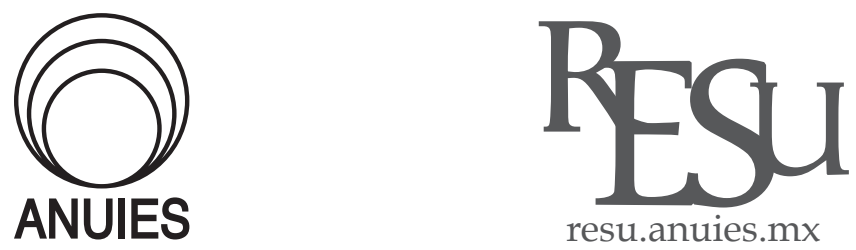

ARTíCULO

\title{
La escritura académica en estudios de ingeniería: valoraciones de estudiantes y profesores*
}

\section{Academic writing in engineering programs: students and teachers assessment}

\begin{abstract}
María Dolores Flores Aguilar**
** Este artículo es producto del financiamiento recibido por parte del Programa de Desarrollo Profesional (Prodep) como resultado de la aplicación a la convocatoria 2013 para profesores de nuevo Tiempo Completo. Agradecimientos:

A la Dra. Montserrat Castelló Badía, investigadora líder de la red europea "European Research Network on Learning to Write Effectively (ERN-LWE)", por su interlocución y guía para el uso de los instrumentos utilizados para recabar la información presentada en el artículo. A la M. en C. Irma Lorena Sánchez Humarán, al Ing. Juan Carlos Franco Ortega y al Ing. Oscar Jesús Guevara Peredia, catedráticos del Tecnológico Nacional de México, Instituto Tecnológico de Mazatlán por su colaboración en la aplicación de los instrumentos y su interlocución.

** Tecnológico Nacional de México. Instituto Tecnológico de Mazatlán. Departamento de Metal Mecánica
\end{abstract} Mazatlán, Sinaloa, México. Correo electrónico: ma.dolores.flores@gmail.com

Recibido el 30 de agosto del 2017; aceptado el 20 de junio del 2018.

\section{Resumen}

Este artículo contribuye a esclarecer la percepción de estudiantes y docentes de dos carreras de ingeniería sobre las prácticas de escritura académica en el aula, su retroalimentación y la evaluación de competencias de escritura en las disciplinas. Se adaptó y aplicó la "Encuesta Europea sobre la Escritura Académica (EEEA)" a una muestra de 86 estudiantes y 15 de sus profesores. Destaca que estudiantes y profesores coincidan en la necesidad de mayor retroalimentación de los textos escritos y que, ante competencias de escritura académica relevantes para los profesores, los estudiantes refieran sentirse inseguros de su dominio.

Palabras clave: Escritura académica; Carrera de ingeniería; Retroalimentación; Comunidad de aprendizaje 


\begin{abstract}
This article contributes to clarify students and teachers perceptions from two engineering programs on writing practices in the classroom, feedback, and assessment of writing competences in the disciplines. The "European Writing Survey (EUWRIT)" was adapted and applied to a sample of 86 students and 15 of their professors. It is important to note that students and teachers agree on the need for greater feedback of written texts and, faced with academic writing skills relevant to teachers, students say they feel insecure about their domain.
\end{abstract}

Keywords: Academic writing; Engineering program; Feedback; Learning community

\title{
Introducción
}

Muchos profesionales de la ciencia piensan que los "buenos" estudiantes encuentran su camino por sus propios medios, mientras que al resto no se les puede ayudar. Esto justifica la negligencia y, tal vez no casualmente, reduce la carga de trabajo. Debe haber algún sentido de selección darwiniana implícita en la "negligencia benigna", pero en un amplio panorama, fallar en enseñar ciencia para sobrevivir resulta en desperdiciar una gran cantidad de talento y tiempo estudiantil y muy frecuentemente convierte en un desorden la vida de los estudiantes (Feibelman, 1993: ix).

$\mathrm{U}$

n proyecto de investigación se plantea cuando no hay respuestas a preguntas que en principio pueden parecer ingenuas, tanto para estudiosos como para no estudiosos del tema en cuestión. El campo de la escritura es uno en los que convergen opiniones diversas que podrían parecer hechos y no lo son. Este artículo aporta hallazgos sobre las percepciones de lo que escriben en el aula los estudiantes de ingeniería y lo que solicitan sus profesores; su importancia radica en que esclarece las diferencias entre ambos colectivos acerca de lo esperado por unos (los profesores) y lo que otros realizan (los alumnos).

Se muestra una parte de los resultados del proyecto de investigación "La elaboración de documentos recepcionales y la comunidad discursiva de 
ingeniería" ${ }^{1}$ realizado entre 2013 y 2016 en una institución universitaria. Uno de los objetivos de este proyecto fue precisamente analizar las percepciones de estudiantes y docentes de ingeniería sobre las prácticas de escritura académica en el aula. Para realizar tal análisis se administraron dos tipos de cuestionarios, uno dirigido a profesores y otro a estudiantes de dos carreras de ingeniería, con base en la Encuesta Europea sobre la Escritura Académica $(\text { EEEA) })^{2}(2015)$.

En un esfuerzo colegiado por tratar de definir las prácticas de escritura académica en ese continente (entre otros objetivos), y con la ayuda de investigadores de cerca de 20 países europeos, se diseñó el instrumento denominado EEEA, que forma parte de los resultados del proyecto "The European Research Network on Learning to Write Effectively", fue previamente probado y sus resultados, si bien arrojan solamente opiniones de estudiantes y sus profesores, son un principio para esclarecer las prácticas de escritura en un contexto universitario específico. A pesar de la gran cantidad de resultados e iniciativas para mejorar las prácticas de escritura académica se puede afirmar lo siguiente:

Sabemos todavía muy poco de cuándo y por qué escriben nuestros estudiantes en diferentes disciplinas, si se les ayuda a gestionar el proceso de composición o sólo se evalúan los textos finales, o si profesores y estudiantes entienden lo mismo cuando solicitan un informe, un resumen o la resolución de un caso por escrito" (Castelló y Mateos, 2015: 492).

El EEEA arroja, por consiguiente, información novedosa sobre las coincidencias y las percepciones de las prácticas de la escritura académica. Su aplicación en España incluyó a nueve universidades y a cuatro áreas del conocimiento, incluída la de ingeniería. Sin embargo, al igual que en México, en España no se han planteado investigaciones longitudinales que puedan dar cuenta del estado general de la escritura académica en las universidades.

La decisión de adoptar este instrumento previamente validado fue gracias a la extensión de su uso en Europa con resultados satisfactorios para identificar las prácticas de escritura en distintas áreas del conocimiento en la

\footnotetext{
${ }^{1}$ Este proyecto recibió financiamiento por parte del Programa de Desarrollo Profesional (Prodep) como resultado de la aplicación a la convocatoria 2013 para profesores de nuevo Tiempo Completo.

${ }^{2}$ Estos cuestionarios son producto de un proceso iniciado en 2006 en la Unión Europea y han sido utilizados de forma diversa (Chitez, Kruse y Castelló, 2015), tanto para evaluar habilidades de escritura, como para comparar la escritura académica en distintas culturas y los géneros en distintos países de Europa del Este.
} 
universidad; se destacaron el rigor con el que fue diseñado y la confiabilidad que arrojaron sus escalas alphas (entre 0.626 y 0.876). En este artículo, sin embargo, solamente se dan los resultados sobre las escalas que muestran las diferencias y las coincidencias que tienen docentes y estudiantes de ingeniería sobre el tipo, el porcentaje, la frecuencia de instrucciones y la retroalimentación del proceso de escritura, además de la autoevaluación y la importancia que le atribuyen a las competencias de escritura académica, lo que se describe un poco más en el apartado de métodos y materiales.

Debido a ello, se consideran relevantes los resultados presentados en este artículo, sobre todo cuando en la actualidad son poco visibles las evidencias de lo que un docente de ingeniería espera que hagan sus estudiantes y lo que efectivamente hacen estos docentes para motivar una escritura adecuada durante los estudios universitarios. Aunque en general los estudiantes esperan cumplir con las expectativas de sus profesores, las prácticas letradas que los anteceden son las únicas que los guían en ocasiones.

Se parte principalmente de dos supuestos: el primero implica que los géneros escritos son constructos sociales "relativamente estables" (Batjín, 2002) que pueden variar con el tiempo, y, por tanto, se requieren referentes compartidos para una comunicación escrita que cumpla con las expectativas de la comunidad discursiva a la que se pertenece; el segundo se relaciona con el concepto de comunidad discursiva (Swales, 1990) que, para designarse como tal, debe contar con objetivos públicos comunes y mecanismos de comunicación entre sus miembros, aprovechar los mecanismos de comunicación establecidos, utilizar uno o más géneros para alcanzar sus objetivos de comunicación discursivos, contar con un léxico específico, además debe estar formado por miembros que tengan un dominio conceptual y la experiencia discursiva mínimos, por lo que se puede decir que, "en estas comunidades, los autores y lectores asumen unos roles predeterminados y son herederos de una misma manera de percibir la realidad y transmitir el conocimiento" (Álvarez y Yániz, 2015: 612).

\section{La competencia comunicativa y la alfabetización académica}

La competencia comunicativa se manifiesta por medio de cuatro habilidades básicas: hablar, escuchar, leer y escribir. Mientras que esta competencia ha sido definida de muy variadas maneras (Hymes, 1972; Maqueo, 2004), se sabe que la escritura es la que media principalmente el aprendizaje en la 
universidad (Halliday y Martin, 1993; Hyland, 2009) y que es en ambientes escolares donde principalmente se aprende a leer y escribir con diferentes fines. Aunque la oralidad continúa prevaleciendo en los ambientes escolares, son las tareas de escritura situada (exámenes, proyectos, diseños, ensayos, prácticas de laboratorio, reportes de campo, notas, por mencionar algunas) las que principalmente sirven para evaluar el conocimiento, independientemente de la asignatura de que se trate y de la tecnología utilizada.

Los enfoques actuales en educación superior han conducido a que los perfiles de egreso de los estudiantes de este nivel incluyan a la competencia comunicativa escrita como una guía en su formación. Esta competencia se refiere principalmente a que los egresados universitarios dominen el modelo de "transformar el conocimiento" (Scardamalia y Bereiter, 1992), que implica la capacidad de construir un texto a través de lo que han dicho otros y expongan sus opiniones sobre ello, más allá de lo que significa reproducir (o transcribir) lo que otros han dicho. Es decir, que en los estudios universitarios se debe privilegiar la escritura epistémica, aquella que le imprime el propio significado a lo escrito, que construye una perspectiva personal a través de lo que alguien ha dicho previamente, más allá de reproducir un tema. De ahí la necesidad de indagar en contextos específicos sobre el significado que le atribuyen docentes y estudiantes a sus prácticas y expectativas de escritura académica situada, y entonces estar en posibilidad de realizar intervenciones educativas que disminuyan las discrepancias que se encuentren.

Se puede entender a la competencia comunicativa como una habilidad que trasciende los ambientes escolares y forma parte de las habilidades de un ser humano para interactuar con el mundo que le rodea de acuerdo a situaciones y contextos específicos, es decir, una persona puede ser competente comunicativamente en ciertos ambientes y en otros no. Por otro lado, la alfabetización académica en los estudios universitarios se enfoca hacia un estudiante que cuente con dichas características más un "conjunto de nociones y estrategias necesarias para participar en la cultura discursiva de las disciplinas así como en las actividades de producción y análisis de textos requeridas para aprender en la universidad" (Carlino, 2005: 13). Como explican Lea y Street (1998), aprender en los estudios universitarios implica la adaptación a nuevas formas de "entender, interpretar y organizar el conocimiento" (2005: 158), mientras que leer y escribir en las disciplinas son procesos por los que los estudiantes se acercan a áreas de estudio desconocidas hasta ahora para ellos. Es preciso afirmar que, de acuerdo con Parodi (2003), "los procesos de alfabetización, tanto inicial como superior, demandan tiempo, esfuerzo y dedicación, pues implican - entre otros- ingresar a una cultura, hacerse parte 
de un grupo y convivir con las normas y regulaciones establecidas en esas determinadas colectividades" además de "respetar las normas que regulan la comunicación especializada en diversos escenarios" (2013: 13).

Por lo antes dicho, un individuo universitario en situaciones generales de aprendizaje necesita aprender formas de comunicación especializada de acuerdo a su disciplina de estudio y el nivel de que se trate. Por lo tanto, quien no cuente con estrategias adecuadas para acercarse, reproducir y reflexionar sobre el conocimiento está en riesgo de quedar excluido de las prácticas profesionales prestigiadas por la comunidad académica a la que desea pertenecer (Ramón y Cajal).

\section{Sobre los procesos de escritura}

Aunque las perspectivas de los actores principales (alumnos y profesores) en los procesos de escritura comenzaron a indagarse recientemente en universidades de habla hispana (Corcelles, Oliva, Castelló y Milian, 2015), han sido poco exploradas en Latinoamérica (Stagnaro y Chosco Díaz, 2013; Carlino, Iglesia y Laxalt, 2013) y en México en particular (Navarro et al., 2016; Barriga Villanueva, 2016; Martínez Compeán, Vélez Espinoza, Ramírez Kano y Pedraza Medina, 2015). Algunos hallazgos relevantes sobre este tema son que el mismo nombre con el que se designa un texto (ensayo, proyecto, reseña, resumen) puede representar distintas concepciones sobre su significado para alumnos y profesores y el que, indistintamente del área del conocimiento de que se trate, la escritura académica universitaria con fines de investigación o de aportación al conocimiento sea la menos utilizada, lo que deja patente que la escritura universitaria responde poco a las demandas actuales de los sistemas educativos universitarios (Corcelles, Oliva, Castelló y Milian, 2015), donde hoy, más que en el siglo pasado, se requiere de un mayor dominio para la gestión de la información y una mayor participación de la cultura escrita.

En investigaciones realizadas en los años ochenta y noventa se comenzaron a diferenciar claramente los procesos que un escritor lleva a cabo cuando produce un texto, antes de estas décadas los estudios se centraban en el análisis de los textos producidos. Desde entonces se ha corroborado que existen diferencias entre los aprendices y los expertos, principalmente en sus procesos de planeación y de autorregulación, en identificar al auditorio al que se dirigen y la situación comunicativa específica de que se trate (Hayes y Flowers, 1983; Scardamalia y Bereiter, 1992; Hayes, 2000).

Debido a que los estudios universitarios, incluidos los de ingeniería, deben dar respuesta a las necesidades de la sociedad actual, es difícil suponer 
que un estudiante de ingeniería quedará exento de ser competente comunicativamente. Dentro de las tareas principales que se espera realice un egresado de ingeniería se encuentran el desarrollo de proyectos tecnológicos, la participación en convocatorias para acceso a recursos económicos y la colaboración en entornos cambiantes. Debido a que estas necesidades implican comunicarse de forma oral y escrita, los organismos acreditadores de programas de ingeniería establecen que los estudiantes muestren su capacidad de comunicarse efectivamente (ABET, 2016; CACEI, 2017), además de que las empresas nacionales y extranjeras ubiquen al dominio de la comunicación escrita como parte de sus requisitos de contratación (Alianza FIIDEM, 2014).

Se tiene la percepción generalizada de que en décadas pasadas los estudiantes escribían mejor que ahora, pero no hay evidencias suficientes para demostrarlo (Barriga Villanueva, 2016; Lunsford y Lunsford, 2008). Sin embargo, sí es posible ubicar diferentes investigaciones que apoyan la necesidad de implementar acciones para mejorar la escritura académica en los estudios universitarios (Velázquez Rivera y Cordóva Jiménez, 2012; Navarro et al., 2016; Moreno de Alba, 2009; Bañales Faz, Vega López, Reyna Valladares, Rodríguez Zamarripa y López Munguía, 2013). Asimismo, desde hace más de tres décadas se ha destacado en el ámbito de la escritura en ingeniería la importancia de identificar la forma en que escriben los estudiantes de esta disciplina y de qué manera afecta a su escritura profesional (Artemeva, 2009).

\section{La investigación sobre la escritura disciplinar en los estudios universitarios}

Desde la década de 1990 se ha señalado que los maestros no están siempre conscientes de las expectativas de escritura, relacionadas con su disciplina, que deben guiar a sus estudiantes. En consecuencia, en algunas ocasiones los estudiantes se enfrentan al silencio o a las explicaciones confusas cuando reciben asesorías para mejorar su escritura académica (Stockton, 1995). En los años recientes, las propuestas de intervención para tratar de mejorar la comunicación escrita se han incrementado, sin embargo, habría que considerar si estas propuestas son resultado de hallazgos de investigación o solamente acciones remediales por la necesidad urgente de resolver la problemática detectada de alfabetización académica en los estudios universitarios.

Aprender a través de textos especializados requiere poner en práctica habilidades cognitivas y de lectoescritura que principalmente provee la comunidad discursiva en la que participa un individuo. Por tanto, la carencia de estas habilidades dificulta el aprendizaje en cualquier disciplina, dada la pri- 
mordial mediación de la lengua escrita en la transmisión de saberes disciplinares. Aún más, si se pretende formar en los estudios universitarios para la vida profesional es imperativo " un manejo eficiente del discurso especializado a través del cual se genera, transmite, disemina y adquiere el conocimiento de cada ámbito" (Parodi, 2004: 8). Empero, hay evidencias de que en los procesos de enseñanza disciplinar escasamente se promueven actividades para dominar los "mecanismos discursivos" (Castelló, 2015: 473) con el objetivo de que el estudiante asimile durante su formación universitaria géneros complejos como el informe o proyecto relacionados con su área de estudio.

Desde hace más de 20 años se ha estudiado la problemática derivada de creer que los estudiantes que llegan a los estudios universitarios no cuentan con el dominio de saberes para escribir adecuadamente (Lea y Street, 1998), este tema ha generado una gran cantidad de resultados en los últimos años (Camps y Castelló, 2013; Bazerman et al., 2012; Carlino, 2005) y ha fortalecido distintas corrientes teóricas, entre ellas el aprendizaje situado (Lave y Wenger, 1991), la teoría del género (Batjín, 2002; Bazerman, 1997) y la Lingüística Sistémico Funcional (Halliday, 1967; Halliday y Hassan, 1989), además de que ha dado pie a discusiones intelectuales complejas por los enfoques empíricos y conceptuales que se han puesto en juego para resolver los desafíos de la escritura académica universitaria (Cisneros Estupiñan y Muñoz Dagua, 2014).

Sin embargo, a pesar de los estudios y corrientes fortalecidas, la investigación sobre los procesos de escritura en español en la universidad, tanto en licenciatura como en posgrado, sigue siendo insuficiente (Agosto-Riera y Mateo-Girona, 2015; Bañales Faz, Castelló Badía y Vega López, 2016; Moreno de Alba, 2009; González Robles, 2014) para dar respuestas concluyentes a preguntas fundamentales tales como: ¿es suficiente con lo aprendido en los anteriores niveles al universitario para ser un comunicador eficaz en la educación superior?; ¿es posible que un estudiante aprenda cómo escribir profesionalmente durante sus estudios universitarios?, o ¿qué enseñar para que los estudiantes aprendan a escribir académicamente? Por lo que aún se necesita esclarecer qué y cómo enseñar a escribir en la universidad de acuerdo con el contexto y la situación de que se trate.

En el estudio denominado "Habilidades lingüísticas de los estudiantes de primer ingreso a las instituciones de educación superior del área metropolitana de la ciudad de México" se afirma que para disminuir la brecha entre la escritura de los estudiantes universitarios y lo que se espera que ellos dominen "resulta crucial que los profesores de todas las asignaturas valoren las tareas de escritura que les solicitan a los alumnos" (Oropeza Gracia, Galán Vélez, Jurado y Vázquez Castro, 2014: 121), lo que es sumamente relevante cuando se sabe que la escritura se da como un proceso situado y específico 
en cada disciplina y asignatura, además de que no es posible transferir completamente las habilidades adquiridas en los niveles educativos anteriores a las exigencias de la universidad.

Se ha demostrado que a través de la mediación de los expertos de un área los aprendices pueden llegar a dominar la cultura y las prácticas propias de una comunidad discursiva de forma más expedita (Lea y Street, 1998; Bazerman y otros, 2012), por lo que la asesoría recibida y el trabajo colaborativo entre estudiantes y docentes para la elaboración de textos disciplinares son acciones requeridas para lograr escritos más aceptables, de manera que conocer las discrepancias entre las percepciones y las expectativas reales puede ayudar a un mejor entendimiento y lograr una mayor y mejor producción de textos.

Es importante destacar que las investigaciones sobre el proceso de escritura académica en los estudios universitarios en México cuentan con escasa tradición (Moreno de Alba, 2009), por lo que se dificulta parcelar los campos sobre el tema o incluso observar continuidad en los eventos que han aportado información sistemática sobre esta área (López Bonilla y Carrasco Altamirano, 2013; Moreno de Alba, 2009). Si bien se pueden ubicar dos enfoques principales en la investigación de la escritura académica (uno desde la lingüística descriptiva y otro desde la lingüística aplicada), distintas tradiciones han guiado principalmente estudios descriptivos y de intervención (Barriga Villanueva, 2016). Asimismo, se encuentran escasamente diferenciados, en las dos corrientes mencionadas, aspectos relativos a las creencias y las prácticas de escritura de docentes y de estudiantes universitarios (Bañales Faz, Vega López, Reyna Valladares, Rodríguez Zamarripa, y López Munguía, 2013; Moreno de Alba, 2009), lo mismo ocurre para el área de ingeniería (Narvaez-Cardona, 2016).

\section{La escritura en los estudios de ingeniería}

La escritura lleva implícita una forma de ver el mundo (Britton, 1970), tanto así que estas miradas permean la perspectiva que el individuo tiene sobre las tareas de aprendizaje. Si se considera que escribir es una práctica cultural, soportada institucionalmente y cuyas funciones principales se aprenden en la escuela, se necesita considerar para su estudio el contexto en el que se produce y las prácticas de enseñanza asociadas (Tolchinsky, 2012). Por tanto, la escritura en cada disciplina está relacionada con los intereses y prácticas propias del campo del conocimiento de que se trate.

A lo largo de una carrera de ingeniería, los estudiantes cursan asignaturas del área de físico-matemáticas, humanidades, ciencias sociales, ciencias de 
la ingeniería y de ingeniería aplicada. En los últimos años de formación se integran los conocimientos adquiridos a lo largo de la carrera y se ponen en juego competencias para plantear proyectos y soluciones a problemas de ingeniería aplicada. Es así que el dominio discursivo para realizar propuestas, fundamentarlas y argumentar sobre su viabilidad requiere tanto de saberes conceptuales propios de la ingeniería como de habilidades retóricas para su planteamiento y no solamente de la capacidad para repetir lo que un libro de texto o un determinado autor manejan sobre el tema.

En los últimos años de estudios es cuando se espera que los alumnos tengan certezas sobre cómo presentar trabajos escritos y exposiciones para convencer a otros sobre sus propuestas. En estas tareas es indispensable que el estudiante maneje con rigor demostraciones matemáticas y aquellas que provienen del área de ciencias que den sustento a sus propuestas. Sin embargo, también sigue siendo un reto en los estudios de ingeniería, al igual que para las clases de ciencias, enseñar a “...establecer relaciones entre informaciones aparentemente dispares y, muy especialmente, a comunicar nuestras ideas e interpretar las expresadas por los demás" (Sanmartí, Izquierdo, y García, 1999: 54), lo cual se aprende particularmente con la práctica, los acuerdos y la interacción que proveen la educación formal y la comunidad discursiva en la que se participa.

Por otro lado, en investigaciones realizadas en Europa y Latinoamérica "los resultados ponen de manifiesto las dificultades de los estudiantes y la ausencia de tareas de escritura epistémica en la educación superior" (Castelló y Mateos, 2015: 16). Al igual que en todas las disciplinas, se necesita que en las carreras de ingeniería se fomente la escritura disciplinar que permita a los estudiantes presentar originalmente innovaciones y diseños de base tecnológica conforme el estado de la técnica de que se trate o lo que previamente se ha diseñado o inventado.

Adicionalmente a lo ya señalado, las prácticas de escritura de quienes estudian ingeniería se encuentran entre perspectivas que en ocasiones difieren entre sí, lo que dificulta aún más su dominio. La visión de los futuros empleadores y las organizaciones de acreditación, por un lado, y los profesores de ingeniería, por otro, pueden tener diferentes exigencias. Mientras que los primeros necesitan que los estudiantes aprendan a realizar propuestas e informes de proyectos para la industria, los segundos requieren que los alumnos realicen prácticas de escritura donde demuestren lo aprendido, con estructuras y formatos escolares (Broadhead, 1999).

Dentro de los criterios que deben cumplir los egresados de ingeniería, se encuentra definido, tanto para México como para otros países, que puedan comunicarse efectivamente, tanto por medios verbales (proyectos, propues- 
tas, contratos, convenios, etc.) como no verbales (planos, diagramas o esquemas); y que puedan colaborar con equipos multidisciplinarios y resolver problemas de ingeniería (ABET, 2016; CACEI, 2017; Proyecto Tuning América Latina, 2007), entre otras competencias que requieren el dominio de la comunicación escrita. En consecuencia, la interacción entre los actores del proceso de enseñanza aprendizaje en las aulas de ingeniería (los estudiantes y sus docentes) debe reflejar actividades y concepciones unificadas sobre los géneros, procesos y prácticas de escritura que un egresado de ingeniería debe haber asimilado durante sus estudios profesionales.

La investigación sobre la escritura de estudiantes de ingeniería en Latinoamérica se considera un campo emergente en los que se podría categorizar, entre otros, escribir para aprender, escribir para prácticas científicas y escribir con fines profesionales (Narvaez-Cardona, 2016). En México no existe un campo de estudio con líneas claras en esta área, además de que presenta distintas interrogantes que abarcan tanto aspectos formales como discursivos. De esta manera, resolver cuestiones tales como si los estudiantes de ingeniería de hoy escriben bien o mal implica adentrarse un poco en las comunidades académicas a las que pertenecen, la manera en que se relacionan para escribir, y los principales géneros que producen para acreditar la formación profesional.

Tal como Parodi (2008) indica, “[...] la escritura en general, y tal vez la escritura académica más aún, es un arduo proceso colectivo y construido a múltiples manos y voces..." (2008: 13), por lo que no se debe perder de vista el análisis de los procesos en los que se construyen documentos escritos colaborativamente, sobre todo cuando en esta producción intervienen asesores y revisores en situaciones diversas. Las evidencias muestran que el desarrollo de la competencia comunicativa escrita depende de altos niveles de autorregulación y del dominio de habilidades de transcripción (Graham y Harris, 2000). Sin embargo, la autorregulación se da una vez que se tiene control sobre lo que se debe regular, de otra manera no hay quien pueda regular lo que no conoce.

Las prácticas de escritura socialmente compartidas (Arciniegas, 2016) son las que más se asemejan a lo realizado en los últimos años de estudios de ingeniería, cuando se presentan propuestas o diseños elaborados por varios alumnos asesorados por especialistas de diversas disciplinas que, a través de distintas iteraciones optimizan un producto académico determinado. Por tal motivo, es indispensable indagar sobre la motivación para escribir y las creencias sobre la propia actuación, la autorregulación y la naturaleza cultural y social de la escritura (Hidi y Boscolo, 2006). 
Como ya fue mencionado, este artículo tiene como objetivo analizar las opiniones de los estudiantes y sus profesores en cuanto a las prácticas de escritura académica en el aula, los apoyos para organizar, retroalimentar y mejorar los textos, la frecuencia y el tipo de retroalimentación, además de la autoevaluación/importancia de las competencias de escritura académica lo largo de los estudios de ingeniería. Todo ello a partir del análisis de la aplicación de la "Encuesta Europea sobre la Escritura Académica" (Chitez, Kruse y Castelló, 2015) en una institución de educación superior mexicana.

\section{Materiales y métodos}

\section{Encuesta Europea sobre Escritura Académica}

La Encuesta Europea sobre Escritura Académica (EEEA o EUWRIT, por sus siglas en inglés) consta de dos cuestionarios, uno dirigido a docentes y otro a estudiantes. Esta encuesta fue probada previamente y mejorada de acuerdo a los primeros resultados (Chitez, Kruse y Castelló, 2015). Estos cuestionarios se encuentran en ocho idiomas y han servido para obtener información sobre las prácticas de escritura académica en la universidad en diversos países. Su aplicación en España durante 2013 arrojó datos que han permitido conocer "cómo estudiantes y profesores describen sus prácticas habituales de escritura y cuáles son las concepciones que sustentan estas prácticas en diferentes contextos disciplinares" (Castelló y Mateos, 2015: 492), lo que permite describir con mayor certeza la comunidad discursiva a la que pertenecen.

Debido a que las investigaciones muestran que para caracterizar las culturas académicas escritas se requieren identificar las prácticas de escritura, las creencias sobre los textos realizados y los procesos para escribirlos, en el diseño de esta encuesta se incluyeron aspectos tales como "tiempo dedicado a la escritura, cantidad y frecuencia de la misma, instrucciones tanto para planificar como para escribir o revisar, tipo de feedback y tipología más frecuente de géneros que escriben los estudiantes", el significado que se le atribuye a planear, escribir y revisar un texto, además de "analizar el tipo de apoyo que los estudiantes desearían recibir para escribir" (Castelló y Mateos, 2015: 494).

Los cuestionarios se probaron en diferentes experiencias y se construyeron a través de diez dimensiones de análisis para estudiantes y sus profesores (Castelló y Mateos, 2015). Estas dimensiones se distribuyen en ocho apartados para estudiantes y seis para profesores, en su mayoría a través de preguntas con escalas de Likert de cinco gradientes. Los instrumentos tienen 
preguntas paralelas para docentes y estudiantes. Los cuestionarios originales $^{3}$ se adaptaron de acuerdo a las características propias del contexto donde se aplicaron. De tal forma que, por ejemplo, se eliminaron cuestionamientos sobre escritura en lengua extranjera (inglés), práctica propia de universidades europeas, que no aplica a la institución donde se realizó el estudio.

El diseño metodológico en el estudio cuyos resultados ahora se presentan se caracteriza como descriptivo, ex post facto y cuantitativo, mientras que el muestreo fue intencional y no probabilístico. En este artículo se presentan los resultados de cuatro escalas: a) prácticas de escritura en el aula (tabla 2); b) apoyo percibido para organizar y retroalimentar y mejorar los textos (tabla 3) y c) frecuencia y tipo de retroalimentación (tabla 4) y evaluación de las competencias de escritura (tabla 5) contenidos en los apartados número dos, tres y cinco de los instrumentos utilizados (Castelló y Mateos, 2015). Se eligieron 24 ítems de estas cuatro escalas, tres sobre las prácticas de escritura, tres sobre los apoyos percibidos, ocho sobre la retroalimentación y diez sobre la evaluación de las competencias de escritura. De acuerdo con el diseño de los instrumentos originales, los ítems para la frecuencia y tipo de retroalimentación solamente están contenidos en el cuestionario para los estudiantes. En la tabla 1 se muestra la distribución de las escalas consideradas en este artículo para su análisis con sus ítems correspondientes.

Tabla 1

Distribución de ítems considerados para estudiantes y profesores

\begin{tabular}{|c|c|}
\hline Escalas e ítems sujetas al análisis & Respuestas \\
\hline Prácticas de escritura en el aula & \multirow{8}{*}{ 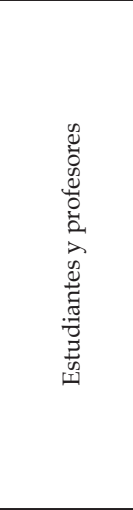 } \\
\hline $\begin{array}{l}\text { 1. ¿Has recibido instrucciones escritas ante una tarea de escritura? / ¿Proporciona } \\
\text { instrucciones escritas para la realización de una tarea de escritura? }\end{array}$ & \\
\hline $\begin{array}{l}\text { 2. ¿Has recibido instrucciones orales ante una tarea de escritura? / ¿Proporciona instrucciones } \\
\text { orales para la realización de una tarea de escritura? }\end{array}$ & \\
\hline $\begin{array}{l}\text { 3. ¿Has debatido con tu profesor sobre tus escritos? / ¿Discute con sus estudiantes sobre sus } \\
\text { textos? }\end{array}$ & \\
\hline Apoyos para organizar, retroalimentar y mejorar los textos & \\
\hline $\begin{array}{l}\text { 1. Los profesores me ayudan a organizar mis textos / La revisión es una parte esencial de la } \\
\text { escritura de los estudiantes. }\end{array}$ & \\
\hline $\begin{array}{l}\text { 2. Recibo suficiente retroalimentación de mis profesores acerca de mis trabajos escritos / Los } \\
\text { estudiantes reciben retroalimentación suficiente sobre sus trabajos escritos. }\end{array}$ & \\
\hline $\begin{array}{l}\text { 3. La respuesta de mis profesores me ayuda a mejorar mi escritura / Los estudiantes } \\
\text { comentan sus textos con alguien y reciben retroalimentación para mejorarlos. }\end{array}$ & \\
\hline
\end{tabular}

Continúa...

${ }^{3}$ Los cuestionarios completos en sus versiones originales se pueden ubicar en los apéndices del monográfico sobre escritura académica publicado en el volumen 27, número 23 de 2015 en la Revista Cultura y Educación. 


\begin{tabular}{|c|c|}
\hline Escalas e ítems sujetas al análisis & Respuestas \\
\hline Frecuencia y tipo de retroalimentación & \multirow{9}{*}{ 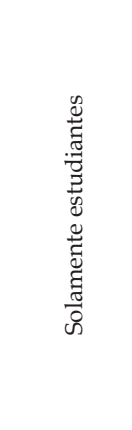 } \\
\hline 1. Retroalimentación del bosquejo o planificación. & \\
\hline 2. Retroalimentación del primer borrador. & \\
\hline 3. Retroalimentación de la versión final. & \\
\hline 4. Únicamente calificación. & \\
\hline 5. Comentarios orales. & \\
\hline 6. Comentarios escritos: generalmente al final del texto. & \\
\hline 7. Comentarios escritos: comentarios en los márgenes. & \\
\hline 8. Calificaciones: puntos valorando diferentes aspectos del texto. & \\
\hline Autoevaluación / Importancia de las competencias de escritura académica & \multirow{11}{*}{ 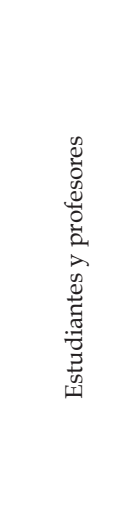 } \\
\hline 1. Comprender y reflexionar acerca de los métodos de investigación. & \\
\hline 2. Encontrar literatura relevante de un tema. & \\
\hline 3. Resumir lecturas. & \\
\hline 4. Planificar el proceso de la escritura. & \\
\hline 5. Organizar y estructurar un documento escrito. & \\
\hline 6. Interpretar e integrar los resultados de investigación. & \\
\hline 7. Argumentar críticamente sobre un tema. & \\
\hline 8. Utilizar la escritura para aprender algo nuevo. & \\
\hline 9. Evaluar el impacto del texto en el lector. & \\
\hline 10. Hacer frente a los problemas surgidos durante proceso escritura & \\
\hline
\end{tabular}

Los ítems elegidos dan cuenta de las prácticas de escritura colaborativa o socialmente compartida (Arciniegas, 2016), en las que intervienen profesores y estudiantes para regular los procesos de escritura en los estudios de ingeniería, lo que da oportunidad de identificar la interacción de la comunidad discursiva de que se trata a lo largo de la carrera.

Aunque las escalas de Likert consideradas para procesar los cuestionarios originales van de 1 a 5 (Castelló y Mateos, 2015), para la codificación de las escalas que ahora se presentan se eligieron entre de 0 a 4 . Para los ítems en el caso de la frecuencia y tipo de instrucciones y retroalimentación las respuestas van de 0 (nunca), hasta 4 (siempre). Para los apoyos de los profesores para organizar, retroalimentar y mejorar los textos se eligió desde 0 , totalmente en desacuerdo, hasta 4, totalmente de acuerdo. En el caso de la evaluación de las competencias de escritura la escala parte de nada seguro/nada relevante (estudiantes/profesores) hasta muy seguro/muy relevante.

Los cuestionarios se aplicaron al término del semestre enero-junio 2015. Se recogieron las opiniones de estudiantes de dos carreras de ingeniería y de sus maestros. Los instrumentos fueron autoadministrados a 86 estudiantes 
(52 de ingeniería mecánica y 34 de ingeniería bioquímica) y 15 profesores (siete de ingeniería mecánica y ocho de ingeniería bioquímica).

Se tomó la decisión de aplicar los instrumentos a dos grupos de estudiantes (uno de cada carrera) que al momento hubiesen superado la mitad de los créditos, ya que se consideró tendrían una visión más general de los procesos de escritura a lo largo de su carrera. Una de las diferencias entre ambas carreras es que una de ellas está más enfocada al desarrollo tecnológico (ingeniería mecánica) y otra hacia las ciencias experimentales (ingeniería bioquímica), además de que la planta docente de la primera tiene menos experiencia en investigación y desarrollo que la segunda.

Asimismo, se solicitó el apoyo a docentes que impartiesen clases en una u otra carrera, en asignaturas del área de ciencias de la ingeniería o ingeniería aplicada y que estuviesen dispuestos a responder los cuestionarios. Una vez aplicados los instrumentos se registró la información en Excel y se exportó al SPSS para su procesamiento estadístico.

\section{Resultados}

De acuerdo con la información recabada en la dimensión de datos personales, se presenta solamente aquélla considerada relevante para este artículo. En el caso de los estudiantes, la muestra estuvo conformada por $73 \%$ de mujeres de ingeniería bioquímica y $27 \%$ de hombres, mientras que todos los estudiantes de ingeniería mecánica fueron del sexo masculino, debido a que en el grupo participante no se encontraba matriculada alguna mujer. Por otro lado, del total de profesores de ambas carreras, al momento de aplicar los instrumentos, $100 \%$ de los profesores participantes de ingeniería bioquímica contaba con estudios de posgrado y se dedicaba a la investigación, mientras que solamente $43 \%$ de los maestros de mecánica tenían estudios de posgrado y su principal función era la docencia. Asimismo, 63\% de los profesores de bioquímica tenía más de 10 años de experiencia docente, mientras que solamente $57 \%$ de los de mecánica contaba con esta antigüedad.

Para el análisis de la fiabilidad del instrumento se utilizó el Alfa de Cronbach y el resultado fue de 0.890 , para el conjunto de 24 ítems analizados, lo que muestra su consistencia interna.

En la tabla 2 se presentan la media, la desviación típica y el nivel de significancia $(p)$ de los tres ítems elegidos en la escala de prácticas de escritura en el aula. En dos de los tres ítems (¿has recibido instrucciones escritas? / ¿Proporciona instrucciones escritas? y ¿has debatido con tu profesor? / ¿Discute 
con sus estudiantes?), se encuentran diferencias significativas, atribuidas a los maestros de bioquímica, en relación con estudiantes de ambas carreras y con profesores de mecánica. En cuanto a recibir instrucciones orales, solamente en promedio, los profesores de bioquímica (3.38) más experimentados por su formación en la producción de artículos de investigación opinan por encima de los estudiantes de ambas carreras y de los profesores de mecánica; aunque estadísticamente no resulta significativa la diferencia, sí se observa un mayor índice también para este ítem en los profesores de bioquímica.

La mayor diferencia entre lo expresado por estudiantes y por profesores de ambas carreras en los ítems presentados en la tabla 2 se centra en la respuesta a la pregunta "¿has debatido con tu profesor sobre tus escritos? / ¿Discute con sus estudiantes sobre sus textos?" , con un nivel de significancia de 0.003 . La mayor discrepancia se encuentra entre los profesores de bioquímica y sus estudiantes, donde los primeros indican que con frecuencia (un promedio de 3 en la escala) discuten con sus estudiantes sobre sus textos, mientras que el promedio de los estudiantes se sitúa en 1.5 (entre raramente y a veces, de acuerdo con la escala señalada). Existe también discrepancia, aunque menor (0.29) en esta pregunta para los promedios de respuesta de estudiantes de mecánica (1.48) y sus profesores (2.17).

Tabla 2

Prácticas de escritura en el aula

\begin{tabular}{|c|c|c|c|c|c|}
\hline & \multicolumn{2}{|c|}{ Estudiantes } & \multicolumn{2}{|c|}{ Profesores } & \\
\hline & Mecánica & Bioquímica & Mecánica & Bioquímica & \\
\hline & \multicolumn{4}{|c|}{$\bar{x}(\mathrm{DT})$} & $p$ \\
\hline $\begin{array}{l}\text { 1. ¿Has recibido instrucciones } \\
\text { escritas ante una tarea de } \\
\text { escritura? / ¿Proporciona } \\
\text { instrucciones escritas para la } \\
\text { realización de una tarea de } \\
\text { escritura? }\end{array}$ & $2.16(1.131)$ & $2.21(1.038)$ & $2.14(.690)$ & $3.50(.756)$ & 0.011 \\
\hline $\begin{array}{l}\text { 2. ¿Has recibido instrucciones } \\
\text { orales ante una tarea de escritura? } \\
\text { / ¿Proporciona instrucciones } \\
\text { orales para la realización de una } \\
\text { tarea de escritura? }\end{array}$ & $2.76(1.061)$ & $2.47(.896)$ & $3.00(.577)$ & 3.38 (1.188) & 0.107 \\
\hline $\begin{array}{l}\text { 3. ¿Has debatido con tu profesor } \\
\text { sobre tus escritos? / ¿Discute con } \\
\text { sus estudiantes sobre sus textos? }\end{array}$ & 1.48 (1.182) & $1.50(1.022)$ & 2.17 (1.169) & $3.00(.926)$ & 0.003 \\
\hline
\end{tabular}

Escala de Likert: $0=$ nunca; 1 = raramente; 2 = a veces; $3=$ con frecuencia; $4=$ siempre

En lo que respecta a los apoyos percibidos para organizar, retroalimentar y mejorar los textos (tabla 3), los profesores de ambas carreras indican no estar seguros (un promedio entre 2.50 y 2.63) en que la revisión es una parte esen- 
cial de la escritura de los estudiantes; mientras que los estudiantes principalmente opinan que en promedio sus profesores no les ayudan a organizar sus textos (0.94 para estudiantes de mecánica y 1.76 para los de bioquímica).

Asimismo, tanto estudiantes y profesores de ambas carreras opinan no estar seguros de que la retroalimentación sea suficiente y de que los estudiantes comenten sus textos y reciban retroalimentación. En la opinión promedio de los estudiantes perciben no estar seguros de si la respuesta de sus profesores les ayuda a mejorar su escritura.

Tabla 3

Apoyo percibido para organizar, retroalimentar y mejorar los textos

\begin{tabular}{|c|c|c|c|c|c|}
\hline & \multicolumn{2}{|c|}{ Estudiantes } & \multicolumn{2}{|c|}{ Profesores } & \\
\hline & Mecánica & Bioquímica & Mecánica & Bioquímica & \\
\hline & \multicolumn{4}{|c|}{$\bar{x}(\mathrm{DT})$} & $p$ \\
\hline $\begin{array}{l}\text { 1. Los profesores me ayudan a } \\
\text { organizar mis textos / La revisión } \\
\text { es una parte esencial de la } \\
\text { escritura de los estudiantes }\end{array}$ & $0.94(1.406)$ & $1.76(1.350)$ & $2.50(1.975)$ & $2.63(1.506)$ & 0.001 \\
\hline $\begin{array}{l}\text { 2. Recibo suficiente } \\
\text { retroalimentación de mis } \\
\text { profesores acerca de mis trabajos } \\
\text { escritos / Los estudiantes reciben } \\
\text { retroalimentación suficiente sobre } \\
\text { sus trabajos escritos }\end{array}$ & $2.15(1.144)$ & 2.38 (1.129) & $2.60(1.517)$ & 2 (1.0239) & 0.662 \\
\hline $\begin{array}{l}\text { 3. La respuesta de mis profesores } \\
\text { me ayuda a mejorar mi escritura } \\
\text { / Los estudiantes comentan } \\
\text { sus textos con alguien y } \\
\text { reciben retroalimentación para } \\
\text { mejorarlos. }\end{array}$ & $2.69(1.164)$ & $2.71(0.906)$ & $1.83(1.169)$ & $2(0.756)$ & 0.098 \\
\hline
\end{tabular}

Al analizar el porcentaje de respuestas sobre recibir suficiente retroalimentación de sus profesores acerca de los trabajos escritos, solamente $34 \%$ de los participantes de ambas carreras están totalmente de acuerdo en que lo reciben, en contraste con $28 \%$ acumulado entre quienes están totalmente en desacuerdo o en desacuerdo, $25 \%$ de quienes no están seguros y $13 \%$ de quienes están de acuerdo. Se esperaría que un porcentaje mayor de los estudiantes participantes hubieran respondido estar totalmente de acuerdo en que la retroalimentación es suficiente al encontrar en sus respuestas sobre la frecuencia y el tipo de retroalimentación que reciben.

Es posible observar en la tabla 4, sobre la frecuencia y el tipo de retroalimentación recibida, que en ninguna de las escalas analizadas las medias de las percepciones de los estudiantes de ambas carreras alcanzan el valor de 3 (con frecuencia) y su grado de acuerdo es muy próximo en la mayoría de las 
aseveraciones. La única diferencia significativa $(p=0.042)$ se ubica en la respuesta a la retroalimentación del primer borrador, que es el valor más bajo en promedio obtenido para los estudiantes de ingeniería bioquímica (1.65, entre raramente y a veces). Como se observa, la retroalimentación de la versión final del documento supera en promedio a la retroalimentación del proceso de planeación del escrito (bosquejo y primer borrador). Por otro lado, para los estudiantes de mecánica el puntaje más alto se da en la retroalimentación de la versión final y para los de bioquímica el que únicamente reciban calificación después de la entrega de un trabajo escrito.

Tabla 4

Frecuencia y tipo de retroalimentación recibida en opinión de estudiantes

\begin{tabular}{lccc}
\hline & \multicolumn{2}{c}{ Mecánica } & \multicolumn{2}{c}{ Bioquímica } \\
\cline { 2 - 4 } & \multicolumn{1}{c}{$\overline{\mathbf{x}}(\mathrm{DT})$} & $p$ \\
\hline 1. Retroalimentación del bosquejo o planificación. & $1.77(1.165)$ & $1.73(0.997)$ & 0.864 \\
\hline 2. Retroalimentación del primer borrador. & $2.13(1.103)$ & $1.65(1.012)$ & 0.042 \\
\hline 3. Retroalimentación de la versión final. & $2.62(1.301)$ & $2.50(1.308)$ & 0.689 \\
\hline 4. Únicamente calificación. & $2.54(1.196)$ & $2.71(1.088)$ & 0.513 \\
\hline 5. Comentarios orales. & $2.56(1.127)$ & $2.69(1.030)$ & 0.598 \\
\hline 6. Comentarios escritos: generalmente al final del texto. & $2.46(1.038)$ & $2.34(1.066)$ & 0.618 \\
\hline 7. Comentarios escritos: comentarios en los márgenes. & $2.06(1.150)$ & $2.18(1.044)$ & 0.626 \\
\hline 8. Calificaciones: puntos valorando diferentes aspectos del texto. & $2.228(1.310)$ & $2.03(1.334)$ & 0.401 \\
\hline
\end{tabular}

Escala de Likert: 0 = nunca; 1 = raramente; 2 = a veces; $3=$ con frecuencia; $4=$ siempre

De la escala de dominio de las competencias de escritura académica se eligieron diez ítems relacionados con el proceso de escritura; asimismo, se identificaron las medias, la desviación típica y el nivel de significancia $(p)$. Por un lado, se preguntó a los estudiantes qué tan seguros se sentían en las competencias de escritura académica y, por el otro, se preguntó a sus profesores qué tan relevante consideraban esas mismas competencias para la escritura académica. Los resultados mostrados en la tabla 5 indican que existe una regularidad en los promedios de las respuestas de los profesores en cuanto al hecho de que consideran relevantes la mayoría de las competencias sobre las que se les preguntó, con excepción de la competencia de resumir lecturas y utilizar la escritura para aprender algo nuevo (para los profesores de bioquímica), y evaluar el impacto del texto en el lector (para los profesores de mecánica). Lo anterior contrasta con las respuestas de los estudiantes en lo que refiere a contar con relativa seguridad al autoevaluar las mismas competencias. Los valores más bajos para los estudiantes de ambas carreras se dieron en el ru- 
bro que corresponde a planificar el proceso de escritura y evaluar el impacto del texto en el lector.

Tabla 5

Autoevaluación / importancia de las competencias de escritura académica

\begin{tabular}{|c|c|c|c|c|c|}
\hline & \multicolumn{2}{|c|}{ Estudiantes } & \multicolumn{2}{|c|}{ Profesores } & \multirow[b]{3}{*}{$p$} \\
\hline & Mecánica & Bioquímica & Mecánica & Bioquímica & \\
\hline & \multicolumn{4}{|c|}{$\bar{x}(D T)$} & \\
\hline $\begin{array}{l}\text { 1. Comprender y reflexionar acerca } \\
\text { de los métodos de investigación. }\end{array}$ & $2.39(1.00)$ & $2.78(0.79)$ & $3.43(0.53)$ & $3.25(0.96)$ & 0.01 \\
\hline $\begin{array}{l}\text { 2. Encontrar literatura relevante de } \\
\text { un tema. }\end{array}$ & $2.41(1.00)$ & $3.00(0.67)$ & $3.29(0.76)$ & $3.63(0.94)$ & 0.00 \\
\hline 3. Resumir lecturas. & $2.66(0.98)$ & $2.69(1.09)$ & $3.17(0.41)$ & $2.88(1.01)$ & 0.67 \\
\hline $\begin{array}{l}\text { 4. Planificar el proceso de la } \\
\text { escritura. }\end{array}$ & $2.18(1.13)$ & $2.29(1.07)$ & $3.33(0.52)$ & $3.63(1.13)$ & 0.00 \\
\hline $\begin{array}{l}\text { 5. Organizar y estructurar un } \\
\text { documento escrito. }\end{array}$ & $2.39(1.06)$ & $2.87(0.88)$ & $3.43(0.53)$ & $3.63(1.02)$ & 0.00 \\
\hline $\begin{array}{l}\text { 6. Interpretar e integrar los } \\
\text { resultados de investigación. }\end{array}$ & $2.59(1.02)$ & $2.63(0.83)$ & $3.67(0.52)$ & $3.88(0.98)$ & 0.00 \\
\hline $\begin{array}{l}\text { 7. Argumentar críticamente sobre } \\
\text { un tema. }\end{array}$ & $2.51(1.01)$ & $2.47(0.84)$ & $3.43(0.79)$ & $3.63(0.97)$ & 0.00 \\
\hline $\begin{array}{l}\text { 8. Utilizar la escritura para } \\
\text { aprender algo nuevo. }\end{array}$ & $2.54(1.01)$ & $2.63(1.01)$ & $3.14(1.07)$ & $2.63(1.04)$ & 0.56 \\
\hline $\begin{array}{l}\text { 9. Evaluar el impacto del texto en } \\
\text { el lector. }\end{array}$ & $2.02(1.09)$ & $2.13(1.07)$ & $2.86(0.90)$ & $3.38(1.11)$ & 0.00 \\
\hline $\begin{array}{l}\text { 10. Hacer frente a los problemas } \\
\text { surgidos durante proceso } \\
\text { escritura. }\end{array}$ & $2.50(1.13)$ & $2.50(0.84)$ & $3.29(1.11)$ & $3.13(1.03)$ & 0.11 \\
\hline
\end{tabular}

\section{Análisis de los resultados}

Cuando se habla de una cultura de la comunicación escrita en la universidad es necesario identificar con la mayor precisión posible las prácticas en la realización de textos académicos que se producen en ese nivel y en las distintas disciplinas, tanto con la intención de aprender como para aportar al conocimiento.

En la educación formal, un escrito se realiza generalmente a solicitud de quien está a cargo de la conducción de un curso, es decir, los textos son escritos por los estudiantes de acuerdo a las pautas de sus profesores. Como se mostró en los resultados, los estudiantes de las dos carreras participantes en el estudio indican una percepción distinta sobre haber recibido instrucciones orales para elaborar sus tareas de escritura por parte de sus profesores, y discutir con ellos sobre los textos escritos. Semejantes resultados fueron en- 
contrados por Álvarez y Yániz (2015) en un estudio donde se aplicó la EEEA en nueve universidades españolas de distintas áreas del conocimiento, incluida la de ingeniería. Habría que considerar tal vez recomendar a los profesores hacer más explícitas y públicas las instrucciones y las intervenciones con sus estudiantes en el proceso de escritura, principalmente si se desea conformar una comunidad discursiva donde los alumnos puedan aprender en mayor medida de la experiencia de sus profesores.

Resulta relevante destacar que tanto profesores como estudiantes afirman no estar seguros de la retroalimentación que dan (los primeros), y que reciben (los segundos) para elaborar trabajos escritos realizados a lo largo de su carrera. Sin embargo, habría que indagar sobre el tipo de retroalimentación a la que se refieren, dado que los mismos profesores respondieron no estar seguros de que la revisión fuese una parte esencial de la escritura, cuando se sabe que sí lo es (Hayes y Flowers, 1983). La revisión es una habilidad discursiva esencial en la escritura académica, tanto por el proceso mismo de alfabetización académica (Carlino, 2005), como por las normas establecidas en la comunicación especializada (Parodi G. , 2013), características de este nivel de estudios.

Se sabe que la revisión y el apoyo para organizar los textos de los estudiantes es una tarea que debe promoverse esencialmente entre la comunidad discursiva a la que pertenecen. Sin embargo, debe recordarse aquí que las respuestas observadas solamente tienen relación con lo que los estudiantes y profesores perciben y tendría que realizarse un estudio más a fondo para contrastarlo con la realidad de las prácticas en el aula, como lo señalan Castelló (2015) y Álvarez y Yániz (2015).

Si se considera que la escritura socialmente compartida (Arciniegas, 2016) es característica de los desarrollos tecnológicos y científicos, el proceso de retroalimentación es una parte esencial para llegar a acuerdos de escritura y formar a los aprendices en una comunidad discursiva determinada. Se requiere, por tanto, mostrar que la retroalimentación es una etapa en que no se debe pasar por alto en la escritura académica de comunidades que realizan trabajos colaborativos. Es necesario recalcar que los estudiantes manifiestan una necesidad de mayor retroalimentación, por lo que, si ésta se incrementa, se contribuiría a mejorar los productos y procesos de escritura de ambos programas.

Puede observarse que tanto los estudiantes de bioquímica como los de mecánica afirman estar más seguros que sus respectivos profesores en que la retroalimentación que reciben les ayuda a mejorar sus escritos, mientras que sus profesores no están seguros de que alguien les apoye para mejorar los mismos. Institucionalmente, habría que hacer mayor difusión de la importancia de involucrarse con los estudiantes para apoyar su formación como 
escritores en sus propias disciplinas, dada la experiencia de cada profesor en su asignatura y el reconocimiento de que todo profesor es un profesor de lengua, ya que son modelos a seguir y el idioma es el vehículo principal por el que se enseña y aprende.

En cuanto a la autoevaluación de las competencias de escritura académica, es posible observar que, de acuerdo con las respuestas a los ítems seleccionados, los estudiantes se perciben a sí mismos como inseguros en aspectos que inciden en la escritura epistémica (o en la construcción del conocimiento), esa escritura que se considera relevante en el nivel universitario porque manifiesta la habilidad de mostrar su propia voz a través de lo que otros han dicho sobre un tema. Al considerar que estas mismas competencias son relevantes para la escritura académica en los estudios de ingeniería, los profesores pueden ser interlocutores y acompañar a sus estudiantes desde la planeación de sus textos hasta la retroalimentación de los mismos. Con ello se estaría integrando a los aprendices en las prácticas expertas de comunicación escrita en la correspondiente área del conocimiento de que se trate.

Asimismo, se esperaba que los estudiantes de la carrera con mayor enfoque experimental (ingeniería bioquímica) tuvieran mayor seguridad en las competencias de escritura académica, pero ninguno de sus promedios obtenidos en la autoevaluación alcanza el estar seguros sobre su dominio.

Como señalan Marín, López, y Roca-De-Larios (2015), se deben “... interpretar los resultados relativos a esta parte de la muestra como fenómenos relacionados con el campo de la opinión más que como una valoración directa de hechos observados a través del contacto con el estudiante y su labor como escritor" (2015: 522). Más adelante se deben contrastar estos resultados con hechos observados de lo que se efectúa en las distintas asignaturas a lo largo de la carrera. Sin embargo, es importante conocer las percepciones sobre las prácticas de escritura, ya que hasta el momento son escasos los datos de este tipo que permitan esclarecer los procesos de comunicación escrita en ingeniería a través de las opiniones de sus principales actores.

\section{Conclusiones}

Se debe hacer énfasis en que, tal como distintos investigadores lo han señalado (Parodi G. , 2008; Carlino, 2005; González Robles, 2014), los estándares reconocidos como aceptables en una determinada área del conocimiento provienen de quienes son líderes en esa comunidad y los aprendices deben ser instruidos para cumplir con sus expectativas, de otra forma se estará jugando a una especie de adivinanza donde los estudiantes se someten a evaluaciones 
de sus tareas de escritura esperando cumplir con lo que se les solicita pero sin tener bases o modelos reales para ello.

Construir el conocimiento a través de lo que los expertos en un campo han publicado es una tarea compleja, que encierra procesos difíciles de adquirir con el solo contacto con textos universitarios. Se necesitan políticas educativas claras sobre alfabetización académica que se concreten en prácticas de aprendizaje reales. Es insuficiente que en los perfiles de egreso de los programas de ingeniería o en las declaraciones y los documentos de instituciones se hable sobre la competencia comunicativa escrita que un ingeniero debe demostrar, si no se concreta en acciones provenientes de investigaciones que vayan más allá de acciones remediales y que se establezcan como programas permanentes semejantes a los ya establecidos en diversos países en Latinoamérica (Narvaez-Cardona, 2016; Carlino, 2013; Castelló, 2014) y el mundo en general.

Si se desea contar con mayor producción científica y tecnológica en las instituciones formadoras de ingenieros, un camino es formarlos como escritores y alfabetizarlos académicamente en sus disciplinas, ya que no fueron preparados para ello en los niveles anteriores. "Este compromiso institucional incluye además una dimensión ética por cuanto alude al necesario equilibrio entre lo que se requiere de los estudiantes y lo que las instituciones están poniendo a su disposición para que puedan conseguirlo" (Castelló, 2015: 472).

Es necesario señalar que los aspectos superficiales de un texto, tales como la ortografía, la estructura y los formatos de un documento son relativamente sencillos de transferir a través de talleres donde se comparten modelos de escritura, sin embargo, aspectos discursivos como jerarquización de ideas, estructuras argumentativas, descriptivas o narrativas, y la cohesión del texto, resultan difíciles de aprender, ya que implican práctica constante, trabajo colaborativo y autorregulación de la escritura. La construcción del conocimiento es difícil de enseñar en cursos aislados, se requiere la práctica y colaboración constante con quienes dominan las formas de "transformar el conocimiento" para contar con elementos de revisión que permitan autorregular la elaboración de un texto situado. Si bien se deben disminuir las discrepancias entre lo señalado en los programas de estudio y las prácticas correspondientes, es necesario realizar acciones puntuales concretas.

\section{Referencias}

ABET (2016). 2017-2018 Criteria for Accrediting Engineering Programs. Baltimore: ABET. Recuperado de http://www.abet.org/wp-content/uploads/2016/12/ E001-17-18-EAC-Criteria-10-29-16-1.pdf, 22 de enero de 2017. 
Agosto-Riera, Silvia-Eva, y Mateo-Girona, María-Teresa (2015). La enseñanza de la escritura académica en la universidad española. Análisis de experiencias y prácticas relevantes. Cultura y Educación, 27 (3), 629-648. doi:10.1080/113564 05.2015.1072355

Alianza FIIDEM (2014). Resultados de la encuesta a empresas nacionales y extranjeras que trabajan en México. México: Alianza FIIDEM.

Álvarez, Manuela y Yániz, Concepción (2015). Writing Practices in Spanish Universities / Las prácticas escritas en la universidad española. Cultura y Educación, 27 (3), 594-628.

Apéndice 1. Encuesta Europea sobre la Escritura Académica (EEEA). Versión estudiantes. (2015). Cultura y Educación, 27 (3), 649-659. doi:10.1080/11356405.2 015.1089067

Apéndice 2. Encuesta Europea sobre la Escritura Académica (EEEA). Versión profesores. (2015). Cultura y Educación, 27 (3), 660-668. doi:10.1080/11356405.201 5.1089069

Arciniegas, Esperanza (2016). La escritura socialmente compartida en el aula universitaria : la autoregulación. Lenguaje, 44 (2), 197-226.

Artemeva, Natasha (2009). Stories of Becoming: A Study of Novice Engineers Learning Genres of Their Profession. En Charles, Bazerman, Adair Bonini, y Debora Figueiredo, Genre in a Changing World (págs. 158-178). Fort Collins, Colorado: The WAC Clearinghouse-Parlor Press. Recuperado de https:/ / wac. colostate.edu/books/genre/chapter8.pdf

Bañales Faz, Gerardo, Castelló Badía, Montserrat y Vega López, Norma A. (2016). Enseñar a leer y escribir en la educación superior. Propuestas educativas basadas en la investigación. México: Fundación SM.

Bañales Faz, Gerardo, Vega López, Norma A., Reyna Valladares, Antonio, Rodríguez Zamarripa, Brianda S., y López Munguía, Ricardo (2013). Investigación de la lectura y la escritura académica en la educación media superior y superior. En A. Ávila, A. Carrasco Altamirano, A. A. Gómez Galindo, M. T. Guerra Ramos, G. López-Bonilla, y J. L. Ramírez Romero, Una década de investigación educativa en conocimientos disciplinares en México. Matemáticas, ciencias naturales, lenguaje y lenguas extranjeras (págs. 327-335). México: ANUIEs-COMIE.

Barriga Villanueva, Rebeca (Coordinadora), con la colaboración de Odette Hernández Cruz. (28 de Septiembre de 2016). Lingmex: Bibliografía Lingüística de México desde 1970. 19a. ed. El Colegio de México. Obtenido de http:// lingmex.colmex.mx/

Batjín, Mijail (2002). El problema de los géneros discursivos. En Estética de la creación verbal. México: Siglo xxI.

Bazerman, Charles (1997). The Life of Genres, the Life in the Classroom. En W. Bishop, y H. Ostrom (Edits.), Genre and Writing: Issues, Arguments, Alternatives (págs. 19-26). Portsmouth: Boynton/Cook.

Bazerman, Charles, Dean, Chris, Early, Jessica., Lunsford, Karen, Null, Suzie, Rogers, Paul y Stansell, Aamanda (2012). International Advances in Writing Research: Cultures, Places, Measures. Fort Collins, Colorado: The WAC Clearinghouse.

Britton, James (1970). Language and Learning. Londons: Allen Lane. 
Broadhead, Glenn (1999). Addressing Multiple Goals for Engineering Writing: the Role of Course-specific Websites. Language and Learning Across the Disciplines, 3 (2), 19-43.

CACEI (2017). Marco de Referencia del CACEI 2018 en el marco internacional. México: CACEI. Recuperado de http://cacei.org.mx/images/Marco_2018/L-CACEIDGE-05_marco_ref_ING_inst_2018_2_08feb17.pdf, el 20 de febrero de 2018,

Camps, Anna, y Castelló, Montserrat (Enero-Abril de 2013). La escritura académica en la universidad. Revista de Docencia Universitaria, 11 (1), 17-36. Recuperado de http:/ / red-u.net/redu/documentos/vol11_n1_completo.pdf

Carlino, Paula (2005). Escribir, leer y aprender en la universidad. Una introducción a la alfabetización académica. Buenos Aires: Fondo de Cultura Económica de Argentina.

Carlino, Paula (2013). Alfabetización académica. Diez años después. Revista Mexicana de Investigación Educativa, xVIII (51), 355-381. Recuperado de http:/ / www.comie.org.mx/v1/revista/visualizador.php?articulo=ART57002ycriter io=http:/ / www.comie.org.mx/documentos/rmie/v18/n057/pdf/57002.pdf

Carlino, Paula, Iglesia, Patricia y Laxalt, Irene (2013). Concepciones y prácticas declaradas de profesores terciarios en torno al leer y escribir en las asignaturas. Revista de Docencia Universitaria, 11 (1), 105-135.

Castelló, Montserrat (2015). Research on Academic Writing in the Teaching Learning Processes in the Spanish University Context / La investigación sobre escritura académica en los procesos de enseñanza- aprendizaje en la universidad española. Cultura y Educación, 27 (3), 465-476.

Castelló, Montserrat y Mateos, Mar (2015). Faculty and Student Representations of Academic Writing at Spanish Universities / Las representaciones de profesores y estudiantes sobre la escritura académica en las universidades españolas. Cultura y Educación, 27 (3), 477-503.

Chitez, Madalina, Kruse, Otto y Castelló, Montserrat (2015). The European Writing Survey: Background,Structure, Implementation, and Some Results. Winterthur: ZHAW Zurich University of Applied Sciences. (Working Papers in Applied Linguistics 9). Recuperado de https://digitalcollection.zhaw.ch/ bitstream/11475/1016/1/407433819.pdf, 10 de Junio de 2017.

Cisneros Estupiñan, M., y Muñoz Dagua, C. (2014). Tras las huellas de las investigaciones sobre lectura y escritura en la universidad. Pereira, Colombia: Universidad Tecnológica de Pereira.

Corcelles, Mariona, Oliva, Àngels., Castelló, Montserrat y Milian, Martha (2015). Writing at University: are we on the Same Page? / Escribir en la universidad: ¿nos entendemos? Cultura y Educación, 27 (3), 534-568. doi:10.1080/11356405 .2015.1072359

Feibelman, Pete J. (1993). A Ph. D. is not Enough. A Guide to Survival in Science. Reading: Addison-Wesley Publishing Company.

González Robles, Rosa O. (2014). Habilidades lingüísticas de los estudiantes de primer ingreso a las instituciones de educación superior del área metropolitana de la ciudad de México. México: ANUIEs. Recuperado de http://asambleaanuies.ibero. $\mathrm{mx} / \mathrm{wp}$-content/uploads/2013/11/Estudio-EXHALING-6-nov.pdf, el 15 de diciembre de 2014. 
Graham, Steve y Harris, Karen R. (8 de Junio de 2000). The Role of Self-Regulation and Transcription Skills in Writing and Writing Development. Educational Psychologist, 35 (1), 3-12. doi:10.1207/S15326985EP3501_2

Halliday, Michael Alexander Kirkwood (1967). Grammar, Society and the Noun. Londons: H. K. Lewis.

Halliday, Michael Alexander Kirkwood y Hassan, Ruqaiya (1989). Language, Context and Text: aspects of Language in a Social-Semiotic Perspective. Oxford: Oxford University Press.

Halliday, Michael Alexander Kirkwood y Martin, J. R. (1993). Writing science: Literacy and discursive power. Pittsburgh: University of Pittsburgh Press.

Hayes, Jhon R. (2000). A New Framework for Understanding Cognition and Affect in Writing. En R. Indrissano, y J. R. Squire, Perspectives on Writing: Research, Theory, and Practice (págs. 6-45). Newark, Estados Unidos: International Reading Association.

Hayes, Jhon R. y Flowers, Linda S. (1983). A Cognitive Model of Writing Process in Adults. Final Report. Carnegie Mellon University. Pittsburgh: ERIC. Obtenido de http:/ / files.eric.ed.gov/fulltext/ED240608.pdf

Hidi, Suzanne y Boscolo, Pietro (2006). Motivation and Writing. En C. A. McArthur, S. Graham, y J. Fitzgerald (Edits.), Handbook of Writing Research (págs. 144-157). New York: Guilford Press.

Hyland, Ken (2009). Academic discourse. English in a Global Context. London: Continuum.

Hymes, Dell (1972). On Communicative Competence. En J. B. Pride, y J. Holmes (Edits.), Sociolinguistics. Selected Readings (págs. 269-293). Harmondsworth: Penguin.

Lave, Jean y Wenger, Ettiene. (1991). Situated Learning: Legitimate Peripheral Participation. Cambridge: Cambridge University Press.

Lea, Mary R. y Street, Brian V. (1998). Student Writing in Higher Education: an Academic Approach. Studies in Higher Education, 23 (2), 157-172.

López Bonilla, Guadalupe y Carrasco Altamirano, Alma (2013). Lenguaje y Educación: una década de investigación. En A. Ávila, A. Carrasco Altamirano, A. A. Gómez Galindo, M. T. Guerra Ramos, G. López-Bonilla, y J. L. Ramírez Romero, Una década de investigación educativa en conocimientos disciplinares en México. Matemáticas, ciencias naturales, lenguaje y lenguas extranjeras (págs. 379418). México: ANUIES-COMIE.

Lunsford, Andrea A. y Lunsford, Karen J. (2008). "Mistakes Are a Fact of Life": A National Comparative Study. College Composition and Communication, 59 (4), 781-806.

Maqueo, Ana María (2004). La competencia comunicativa y el enfoque comunicativo funcional. En A. M. Maqueo, Lengua, aprendizaje y enseñanza (págs. 147-205). México: Limusa.

Marín, Javier, López, Sonia y Roca-De-Larios, Julio (2015). The Academic Writing Process in Spanish Universities: Perceptions of Students and Faculty / El proceso de escritura académica en la universidad española: percepciones de estudiantes y profesores. Cultura y Educación, 27 (3), 504-533. 
Martínez Compeán, Ma. Eugenia, Vélez Espinoza, Maricela, Ramírez Kano, Efrén y Pedraza Medina, Haydée (2015). Las creencias de docentes universitarios sobre las prácticas de lectura y escritura. En E. Ramírez Leyva, Tendencias de la lectura en la universidad (págs. 75-91). México: unAM.

Moreno de Alba, José G. (2009). Historia y presente de la enseñanza del español en México. México: Universidad Nacional Autónoma de México.

Narváez-Cardona, Elizabeth (2016). Latin-American Writing Initiatives in Engineering from Spanish-speaking Countries. Ilha do Desterro, 69 (3), 223-248.

Navarro, Federico, Ávila Reyes, Natalia, Tapia-Ladino, Mónica, Cristovao, Vera L., Moris, María E., Narváez Cardona, Elizabeth y Bazerman, Charles (2016). Panorama histórico y contrastivo de los estudios sobre lectura y escritura en educación superior publicados en América Latina. Revista Signos: Estudios de Lingüística, 49 (S1), 100-126.

Oropeza Gracia, Viviana, Galán Vélez, Rosa Margarita, Jurado, Martha, y Vázquez Castro, Violeta (2014). La expresión escrita y su pauta de corrección. En R. O. González Robles, Habilidades lingüísticas de los estudiantes de primer ingreso a las instituciones de educación superior del área metropolitana de la ciudad de México (págs. 119-222). México: ANUIEs. Recuperado de http://asambleaanuies.ibero. $\mathrm{mx} /$ wp-content/uploads/2013/11/Estudio-EXHALING-6-nov.pdf

Parodi, Giovanni (2004). Textos de especialidad y comunidades discursivas técnico-profesionales: una aproximación basada en corpus. Estudios Filológicos, Septiembre (39), 7-36.

Parodi, Geovanni (2008). Géneros del discurso escrito: Hacia una concepción integral desde una perspectiva sociocognitiva. En Géneros Académicos y Géneros Profesionales: Accesos discursivos para saber y hacer (págs. 17-37). Valparaíso: Ediciones Universitarias de Valparaíso.

Parodi, Geovanni. (2013). Prólogo. En E. Moyano, Aprender Ciencias y Humanidades: una cuestión de lectura y escritura. Aportes para la construcción de un programa de inclusión social a través de la educación lingüística (págs. 13-16). Los Polvorines, Argentina: Universidad Nacional de General Sarmiento. Recuperado de http:/ / www.ungs.edu.ar/cm/uploaded_files/publicaciones/598_EDU13\%20-\%20 Aprender\%20ciencias\%20y\%20humanidades\%20-\%20Web.pdf

Proyecto Tuning América Latina (2007). Reflexiones y perspectivas de la Educación Superior. Informe Final-Proyecto Tuning-América Latina 2004-2007. (P. Beneitone, C. Esquetini, J. González, M. Marty Maletá, G. Siufi, y R. Wagenaar, Edits.) Bilbao, España: Universidad de Deusto.

Ramón y Cajal, Santiago (s. f.). Fundamentos racionales y condiciones técnicas de la investigación biológica. Cajal y la Academia de Ciencias. España: Real Academia de Ciencias. Recuperado el 16 de Mayo de 2015, de http:/ / www.rac.es / ficheros/doc/00207.pdf

Sanmartí, Neus, Izquierdo, Mercé y García, Pilar (1999). Hablar y escribir. Una condición necesaria para aprender ciencias. Cuadernos de Pedagogía (281), 54-58.

Scardamalia, Marlene y Bereiter, Carl (1992). Dos modelos explicativos de los procesos de composición escrita. Infancia y aprendizaje (58), 53-64.

Stagnaro, Daniela y Chosco Díaz, Cecilia (2013). Discordancias entre expectativas docentes y representaciones de los estudiantes en torno a las tareas de escritura académica: diagnóstico y algunas propuestas de intervención. En S. Agoff, 
N. Bengochea, F. Chiodi, C. Chosco Díaz, M. Muschietti, F. Navarro, C. Zunino, y L. Natale (Eds.), El semillero de la escritura. Las tareas escritas a lo largo de tres carreras de la UNGS (págs. 95-128). Los Polvorines, Argentina: Universidad Nacional de General Sarmiento.

Stockton, Sharon (Enero de 1995). Written in History: Narrating the Subject of Time. Written Communication, 12 (1), 47-73.

Swales, Jhon. (1990). The Concept of Discourse Community. En Genre Analysis: English in Academic and Research Settings (págs. 21-32). Boston: Cambridge University Press.

Tolchinsky, Liliana (2012). Introduction: Writing Development. En M. Torrance, D. Alamargot, M. Castelló, F. Ganier, O. Kruse, A. Mangen, L. Van Waes, Learning to Write Effectively: Current Trends in European Research (págs. 3-6). Bingley: Emerald Group.

Velázquez Rivera, Marisol y Cordóva Jiménez, Alejandro (2012). Representaciones sociales de profesores de un programa de Licenciatura en Bioquímica acerca de la enseñanza y aprendizaje de la escritura en la formación académica. Literatura y Lingüística (25), 169. 
